We identified a wide, four point (1-5) variability in satisfaction. Less satisfied NOK predominantly reported reduced frequency of medical communication.

We used PDSA methodology and introduced three interventions: 1) 'Gold standard' for frequency of routine medical updates; 2) Record date of most recent NOK update on the doctors' list; 3) Disseminate a light-hearted informative video of the 'gold standard' to increase awareness and motivation.

Early post-intervention data showed reduced variability in satisfaction, with levels consistently reported as 4 or 5 towards the end of data collection. Process measures demonstrated excellent uptake of interventions with $81.3 \%$ adherence to the 'gold standard' and $95.7 \%$ compliance to accurately updating the doctors' list.

Early data indicates a promising tool for improving doctorNOK communication primarily by prompting doctors to update NOK more regularly.

Our timeline was very limited but the excellent uptake of interventions suggests a potential for sustainable improvement. The lack of defined protocols and openness to rapid change at the NNW encouraged us as junior doctors to take the initiative and lead quality improvement work.

\section{CHANGE TO REDUCE PATIENT WAITING TIMES}

Charlotte Elliott. Countess of Chester Hospital, UK

\subsection{6/leader-2020-FMLM.87}

Introduction Ambulatory Majors was set up in the Countess of Chester Hospitals Emergency Department to take the workstream of 'non minor injury' patients, who are not suitable for the Urgent Treatment Centre and do not need a bed to wait on. Ambulatory Majors has the largest throughput of patients, but has minimal senior doctor supervision. Patients have long waits to be seen with their consultation time disproportionately shorter. Patients are having cannulation or blood tests which are not required which increases patient length of stay. Their length of stay is the biggest factor causing congestion which leads to the impression of chaos to both patients and staff.

Methods The aim of this project was to decrease the length of time from patients' arrival to being seen and patients' arrival to depart. A force field analysis, Driver diagram and a literature search are used to help identify possible solutions to the problem. Buy in was gained from key stakeholders.

Intervention A team-based approach to seeing patients in Ambulatory Majors was adapted which included two doctors and a nurse seeing each patient. Whilst one doctor saw the patient, the other doctor recorded the notes in real time and the nurse preformed any investigations necessary.

Results The average arrival to seen improved from an average of 139.63 minutes to 112.5 minutes during the first month and average arrival to departure time from an average of 194 minutes to 162.5 minutes. Results over the year saw an initial decrease for the month of March when the intervention was introduced but this was not sustained throughout the year.

Conclusion This project explored ways of reducing time to be seen and the length of overall stay in ambulatory majors. Strategies using alternative team working and environmental change were used. Despite some promising initial results, benefits were not sustained which were multifactorial.
Leading innovation and improvement

\section{INFORMATION GIVEN POST DIAGNOSIS OF AUTISM: OBTAINING PARENT FEEDBACK TO IMPROVE EXISTING PRACTICE}

Dannika Buckley*, Charlotte Pay, Naomi Elson, L Killian. Western Sussex Hospitals NHS Trust, UK

10.1136/leader-2020-FMLM.88

Aim To gain feedback from parents of children diagnosed with an

Autistic Spectrum Condition regarding information they received following diagnosis, with a view to improve our service. Method Feedback was initially sought using a questionnaire that was distributed to all parents of children with a new diagnosis of autism by consultants at Worthing Child Development Centre (CDC), during a 6 month period. After a very low response rate, a focus group was arranged by inviting 20 parents, randomly selected from all those diagnosed in a 4 month period. Children with dual diagnoses and parents unable to speak English without an interpreter were excluded. There were 3 facilitators and 1 scribe.

Results 4 parents attended the focus group. Parents preferred diagnostic information given to them by a Speech and Language Therapist alongside their consultant. They found it difficult to take in the information and would have liked written information to take home. A follow up visit from a specialist health visitor was very useful and was felt best at 3-4 weeks post diagnosis. Parents wanted clinic reports to contain information specific to their child, rather than general implications of diagnosis. Social media support groups were named as more useful than official websites.

Conclusion This qualitative study suggests positive aspects of post diagnosis information provided by Worthing CDC, however parents want written information provided sooner. Parents also value social media groups that are not regulated, making their recommendation controversial. Most importantly, although it can be difficult to obtain, parent feedback can provide valuable information to ensure services best meet the needs of their users and therefore must be sought routinely.

\section{Babylon GP at Hand is an NHS GP practice which predominantly uses remote video or telephone consultations for provision of healthcare; and has done for the last 6 years. Various clinicians are available including GPs, prescribing pharmacists and advanced}

\section{CONDUCTING REMOTE ASTHMA REVIEWS TO INCREASE ACCESSIBILITY: BEST PRACTICE}

Nabila Chaudhri*, Alison Cooper, Farah Haque. Babylon GP at Hand

10.1136/leader-2020-FMLM.89

At Babylon GP at Hand we have been providing asthma reviews via telephone and video initially. A search enables us 
to highlight patients due a review; those patients are invited to book a digital appointment. Follow-up messages \& other methods are used to engage 'non-responders'.

Increased accessibility Appointments are made by patients through an app and conducted remotely $\&$ available also during evenings \& weekends to increase accessibility.

Use of Clinical Pharmacists

Asthma reviews are mainly conducted by clinical pharmacists ( $\&$ independent prescribers). They are trained to monitor, optimise \& prescribe treatment.

Reviews include monitoring symptoms, inhaler technique, triggers, smoking status, hospital admissions and provision of personalised asthma action plans.

Pharmacists can refer patients to GP's, where they are complex.

Empowering patients Consultation notes are documented in a 'patient friendly' manner and shared with patients via the app. Safety netting, including, information such as 'what to do during an asthma attack' is easily accessible. Asthma actions plans are also emailed to patients. More accessible information empowers the patient to take greater ownership of their health and condition.

Physical follow-up Pharmacists and other healthcare professionals can refer patients for a physical follow up if necessary. This can include a chest examination in an acute exacerbation or a referral for an objective test such as spirometry. Patients can be referred to one of five locations at a convenient date, time and location via an automated link in the app.

Pharmacists can prescribe or ask the patient to purchase a peak flow meter to minimize risk. They are also able to provide written instructions in the app on how to measure and record measurements.

We will be reviewing outcomes of all patients suffering from asthma closely over the next 12 months and obtaining feedback from patients and clinicians involved in their care.

\section{REDUCTION OF INAPPROPRIATE ANTIBIOTIC PRESCRIBING IN A GP PRACTICE LED BY PRACTICE PHARMACISTS}

Farah Haque*, Nabila Chaudhri. Babylon GP at Hand, UK

10.1136/leader-2020-FMLM.90

Aim Establish:

- The proportion of total volume of antibiotics*

- The proportion of broad spectrum* antibiotics prescribed

- Ensure local targets were met (Please see results) despite a growing list size

- To promote antibiotic stewardship

Methods CCG data on antibiotic prescribing was reviewed at regular intervals. Baseline data was taken from the period of July 2018 to December 2018. The CCG used EPACT data and adjusted per 1000 STARPU.

The following actions were then taken as a result of this data:

- Antibiotic prescriptions were monitored on a weekly basis against NICE antibiotics guidelines.
- Prescriptions outside of this guidance were reviewed further for appropriateness.

- Learnings were shared with individual prescribers \& the wider team (there were approximately 100 prescribers at the practice in November 2018)

- Discouraging delayed antibiotic prescribing (improved access means patients are able to book subsequent appointments easily if necessary)

These actions were driven by two practice pharmacists.

Results Quantity of co-amoxiclav, cephalosporin and quinolone items*: The quantity reduced by 9 points* $(36 \%) \quad(\mathrm{p}<0.001)$ (Target $<40$ )

Quantity of total antibacterials*: The quantity reduced by $131 *(30 \%)(\mathrm{p}<0.001)($ Target $<350)$

*Quantity per 1000 antibacterial STAR PU (From Hammersmith and Fulham CCG data)

Discussion/conclusion All results were per 1000 registered users and were STAR PU adjusted (specific therapeutic agesex related prescribing unit) allowing us to compare with other practices in the locality. Monitoring of antibiotics and sharing learnings on an ongoing basis by practice pharmacists has made a statistically significant impact on reducing the number of antibiotics prescribed and so assisted in antibiotic stewardship. Based on this we are sharing the learnings with our practices in Rwanda and Canada with an aim to safeguard antibiotic stewardship globally.

\section{THE FELLOWS UNIT: FORMING A UNIQUE, PRODUCTIVE TEAM DURING A NATIONAL HEALTH EMERGENCY}

${ }^{1}$ Peter Scolding*, ${ }^{2}$ Emma Roche, ${ }^{3}$ Garry Davenport, ${ }^{4}$ Rebecca Rohrer. ${ }^{1}$ NHS England and Improvement, UK; ${ }^{2}$ Birmingham Children's Hospital, UK; ${ }^{3}$ Macmillan Cancer Support, UKi ${ }^{4} B U P A, U K$

\subsection{6/leader-2020-FMLM.91}

Aims The 'Fellows Unit' was set up at NHS England \& Improvement during COVID-19's first wave, consisting of over 20 Clinical Leadership fellows (doctors, dentists and pharmacists) from the Faculty of Medical Leadership \& Management. It aimed to become a novel, cohesive, productive team working on clinical and operational policy in a context of high pace, crisis and uncertainty.

Methods Foundational workshops were held to review progress, to identify the group's unique skill set and professional ethos, to assess challenges and opportunities, and to agree structures and strategies promoting productive working. Bespoke team activities were developed iteratively based on Agile techniques and clinical environment-derived ways-ofworking including:

- Morning stand-up - 20-min digital board round with physical and virtual attendees leading updates on progress, priorities and blockers, based on the Daily Scrum model and clinical board rounds

- MDT allocation meeting - using clinical, behavioural science, project- and knowledge management skillsets to screen a high volume and variety of requests

- Afternoon ABC huddle - well-being and team-building intervention to Appreciate positives, offer suggestions where 